\title{
Modified Criteria for Radiographic Response Assessment in Glioblastoma Clinical Trials
}

\author{
Benjamin M. Ellingson ${ }^{1,2,3,5}$ • Patrick Y. Wen ${ }^{4}$ Timothy F. Cloughesy ${ }^{5,6}$
}

Published online: 20 January 2017

(C) The Author(s) 2017. This article is published with open access at Springerlink.com

\begin{abstract}
Radiographic endpoints including response and progression are important for the evaluation of new glioblastoma therapies. The current RANO criteria was developed to overcome many of the challenges identified with previous guidelines for response assessment, however, significant challenges and limitations remain. The current recommendations build on the strengths of the current RANO criteria, while addressing many of these limitations. Modifications to the current RANO criteria include suggestions for volumetric response evaluation, use contrast enhanced T1 subtraction maps to increase lesion conspicuity, removal of qualitative nonenhancing tumor assessment requirements, use of the postradiation time point as the baseline for newly diagnosed glioblastoma response assessment, and "treatment-agnostic" response assessment rubrics for identifying pseudoprogression,
\end{abstract}

Benjamin M. Ellingson

bellingson@mednet.ucla.edu

1 UCLA Brain Tumor Imaging Laboratory, Center for Computer Vision and Imaging Biomarkers, University of California Los Angeles, 924 Westwood Blvd., Suite 615, Los Angeles, CA 90024, USA

2 Department of Radiological Sciences, University of California Los Angeles, Los Angeles, CA, USA

3 Department of Psychiatry and Biobehavioral Sciences, David Geffen School of Medicine, University of California Los Angeles, Los Angeles, CA, USA

4 Center for Neuro-Oncology, Dana-Farber/Brigham and Women's Cancer Center, Harvard Medical School, Boston, MA, USA

5 UCLA Neuro-Oncology Program, University of California Los Angeles, Los Angeles, CA, USA

6 Department of Neurology, David Geffen School of Medicine, University of California Los Angeles, Los Angeles, CA, USA pseudoresponse, and a confirmed durable response in newly diagnosed and recurrent glioblastoma trials.

Keywords Glioblastoma · GBM · Response Assessment · T1 Subtraction $\cdot$ RANO

\section{Introduction}

Approximately 89,000 new primary brain tumors are diagnosed in the United States each year, for which $27 \%$ are gliomas and $32.8 \%$ are malignant [1]. Glioblastoma (GBM) occurs in approximately $46 \%$ of gliomas [1] and has a poor prognosis of around 14 months median survival [2] and less than $10 \%$ of patients live longer than 5 years from diagnosis [3]. The current standard of care for newly diagnosed GBM patients consists of maximum safe surgical resection followed by external beam radiation therapy plus concomitant and adjuvant temozolomide [2], particularly in patients that demonstrate O6-methylguanine-methyltransferase (MGMT) promoter methylation. At recurrence there is no consensus as to the standard of care as no therapeutic options have produced substantial survival benefit [4].

Although overall survival (OS) is the standard for determining GBM treatment efficacy, using OS as an endpoint when studying new therapeutic strategies can be problematic because of potential influence of therapies prior to or subsequently following the therapy being studied. For example, it is difficult to definitively conclude that bevacizumab has no efficacy in GBM when a large percentage of patients in the placebo arms in both III trials studying efficacy of bevacizumab (i.e. AVAglio and RTOG-0825) eventually crossed over and received bevacizumab (31\% in AVAglio [5] and 48\% in RTOG-0825 [6]). If bevacizumab increased OS when given at any time during treatment, we may expect 
both treatment arms to have similar median OS since most patients eventually were treated with bevacizumab, disguising any therapeutic effects of the drug. Together, these results suggest OS may not be a suitable endpoint when studying new therapeutics or when there is a high chance of cross over in the control arm.

To overcome the limitations associated with using OS as the primary endpoint in studies involving new therapeutics, progression-free survival (PFS) and objective response rate (ORR) should be considered important end points [7]. However, PFS and ORR also have challenges, as determination of response and progression using anatomic imaging techniques may suffer from issues associated with measurement variability and discordance in interpretation between radiologists [8]. Therefore, it is important to develop both new response guidelines for identifying these issues as well as new imaging tools for better differentiating treatment-related changes from changes associated with non-responsive, growing tumor.

The goal of this modified response criteria is to meaningfully evaluate radiographic response and progression while simultaneously allowing therapies that may have transient effects on contrast enhancement but therapeutic benefit to be treated equally. This is particularly important in the context of platform trials, where many different therapies may be compared against a common control and there is a significant risk of over or under estimating tumor burden with a single evaluation time point. By allowing patients to stay on therapy longer, a more comprehensive and accurate assessment of therapeutic benefit can be performed on retrospective examination. A universal set of principles and guidelines, rather than treatmentspecific response criteria, may allow us to fully understand the possible therapeutic benefits and potential limitations of promising new therapies for patients with GBM.

\section{Brief History of Radiologic Response Assessment in GBM}

The formation of new blood vessels, or angiogenesis, is critical for the growth of malignant brain tumors [9-11]. Malignant gliomas with high neovascularity or vascular permeability [12-14] are often associated with higher proliferation rates [15] and higher degree of aggressivity. Because of this association, imaging techniques aimed at identifying abnormal vascularity or vascular permeability, including contrast-enhanced computed tomography (CT) and magnetic resonance imaging (MRI) are commonly used for diagnosis and clinical management of brain tumors, as they have been shown to contain the most aggressive portions of the tumor $[16,17]$.

In 1990, Macdonald et al. [18] introduced the first radiographic response assessment specific to brain tumors by significantly improving upon the Levin criteria [19] and the WHO oncology response criteria [20]. By standardizing the definition of radiographic response using quantitative bidirectional measurements and accounting for corticosteroid use in neurological status, similar to the response evaluation criteria in solid tumors (RECIST) [21], the new "Macdonald criteria" utilized measurements of contrast enhancing tumor size combined with other clinical metrics to determine treatment response and tumor progression by stratifying response into four categories: complete response (CR), partial response (PR), stable disease (SD), and progressive disease (PD). The original Macdonald criteria continues to be the fundamental framework for response assessment and radiographic interpretation of treatment changes in neuro-oncology, having been used for more than 20 years.

\section{Known Limitations for Current Response Criteria}

Although contrast enhancement has been used to assess brain tumor response for more than 60 years and contrast enhancement is generally a strong surrogate of brain tumor disease, there are caveats and exceptions that have been discovered as a result of different treatment mechanisms that affect vascular permeability. For example, increased vascular permeability from cytotoxic therapies including radiotherapy and anti-neoplastic treatments have been shown to result in increased contrast enhancement in the context of therapeutic benefit, a phenomena known as "pseudoprogression." Additionally, clinical studies examining the efficacy of new anti-angiogenic agents have noticed a substantial decrease in contrast enhancement [22-31] resulting in high response rates, ranging from 28 to $63 \%$ in bevacizumab [32-34] and 50\% in cediranib [31] compared with $<10 \%$ using other chemotherapies [35-38], which translated into prolonged PFS but no difference in OS [31, 32]. It was assumed this high response rate was due to the use of contrast enhancement as the primary tool for evaluation in the Macdonald criteria, which resulted in a "pseudoresponse"[39], where contrast enhancement is falsely reduced due to changes in vascular permeability independent of anti-tumor effect.

In addition to increased response rates, studies examining tumor relapse/progression while on anti-angiogenic agents note a tendency for growth of nonenhancing, infiltrative tumor prior to emergence of contrast enhancement [25]. Approximately $30-40 \%$ of patients are estimated to experience non-enhancing tumor progression prior to changes in contrast enhancement [40, 41]. Malignant gliomas are known to contain proportions of both neovascularized and infiltrative tumor [42, 43] and the relative proportions are thought to reflect different biological phenotypes [44-48]. In 2010, expert opinion and examination of these limitations resulted in 
the creation of a formal Response Assessment in NeuroOncology (RANO) criteria [49] to comprehensively reform the Macdonald criteria using previously documented perspectives and approaches [50-52].

Although the RANO criteria corrects for a number of insufficiencies identified in the Macdonald criteria including inclusion of the evaluation of nonenhancing tumor progression and issues associated with pseudoresponse and pseudoprogression, there remain significant limitations to the current standard RANO criteria given recent data. For example, the current RANO criteria requires use of bidirectional measurements of contrast enhancing tumor size, which have been shown to overestimate tumor volume [53] and result in higher reader discordance [8, 54-59], presumably due to differences in head tilt and accurate identification of longest and perpendicular diameter in relatively irregular tumors. Other studies have shown reasonable agreement between bidimensional and volumetric measurements [60,61], suggesting quick bidimensional assessment of contrast enhancing tumor size may be a practical alternative to more sophisticated volumetric segmentation. Additionally, the thresholds used to define response and progression is relatively arbitrary and not optimized based on scientific data showing the best correlation with survival benefit or time to treatment failure. (Note: The efficacy of these thresholds remains to be sufficiently challenged). Also, the use of thresholds based on "percentage change" with respect to baseline tumor size are significantly biased toward small tumors where relatively low absolute changes in tumor size are interpreted as a large percentage change [61]. This is particularly an issue in newly diagnosed GBM studies, where patients with tiny tumors often progress early due to triggering of progression (PD) when "non-measurable disease", defined as having the two largest perpendicular diameters of a contrast enhancing target lesion less than $10 \mathrm{~mm}$, reaches the subtle threshold of "measurable disease". Lastly, although changes in non-enhancing disease were added to the RANO criteria in an attempt to identify non-enhancing tumor progression, particularly in the presence of anti-angiogenic therapy, retrospective evaluations in clinical trials have shown it results in PD approximately a month prior to contrast enhancing disease progression [62], does not result in significant differences in prediction of OS $[62,63]$, and is one of the most controversial aspects of RANO evaluation due to the subjective nature of the interpretation and high adjudication rates. Further, studies have shown that specific aspects of non-enhancing tumor progression (e.g. circumscribed vs. infiltrative $\mathrm{T} 2$ changes) result in dramatically different post-progression survival in GBM patients [41], suggesting evaluation of non-enhancing tumor progression using $\mathrm{T} 2$ and/or FLAIR may be more complex than once thought and warrant further investigation before it can be properly integrated as an early radiographic endpoint. Further, new immunotherapy agents can also cause inflammation leading to changes in T2 signal intensity that is ambiguous with regard to interpretation of changes in tumor biology.

\section{Updated Strategies for Response Assessment in Neuro-Oncology: Modified RANO Criteria}

Based on these various challenges, an update to the current response criteria is necessary in an attempt to establish a general framework for response assessment in neuro-oncology that is agnostic to the mechanism of action of the particular therapy (e.g. anti-angiogenic, anti-neoplastic, immunotherapy, etc.), each of which has its own challenges associated with interpretation of radiographic changes, and is updated based on recent scientific evidence and current clinical convention. In order to advance the RANO criteria and address these challenges we propose the following "modified" RANO criteria for use in evaluating therapeutic efficacy in patients with GBM.

\section{Image Acquisition Requirements}

In response to a need for better standardization of image acquisition in GBM clinical trials [64], a recent consensus paper was published outlining an "international brain tumor imaging protocol (BTIP)" (Table 1) with recommended sequences and parameters [65]. At the core of this recommended protocol is parameter matched, preand post-contrast $3 \mathrm{D}$ (volumetric) inversion recovery gradient recalled echo (IR-GRE) images with less than 1.5$\mathrm{mm}$ isotropic resolution, which allows for both bidimensional and volumetric measurements of enhancing tumor. When possible, this protocol should be employed for prospective clinical trials.

If volumetric acquisition is not employed, or if retrospective evaluations of existing trial data are performed, then slice thickness plus interslice gap should be less than $5 \mathrm{~mm}$. If the sum of the slice thickness and gap exceeds $5 \mathrm{~mm}$, then slightly modified definitions of measurable disease should be used (e.g. measurable disease $=$ largest perpendicular diameters $>2 \times$ slice thickness + gap).

\section{Contrast Enhanced $\mathbf{T}_{1}$-Weighted Digital Subtraction Maps for Increased Lesion Conspicuity}

Quantification of contrast enhancing tumor size or volume should be performed on contrast-enhanced $\mathrm{T}_{1}$-weighted digital subtraction maps (Fig. 1) in order to increase lesion conspicuity and better predict tumor burden in the presence of reduced vascular permeability as occurs during antiangiogenic therapy [66] and/or $T_{1}$ shortening from blood 
Table 1 International Standardized Brain Tumor Imaging Protocol (BTIP) minimum image acquisition requirements for 1.5T and 3T MR systems

\begin{tabular}{|c|c|c|c|c|c|c|}
\hline Variable & 3D T1w Pre ${ }^{b}$ & Ax 2D FLAIR ${ }^{\mathrm{j}}$ & Ax 2D DWI & $\begin{array}{l}\text { Contrast } \\
\text { Injection }^{\mathrm{a}}\end{array}$ & $A x 2 D T 2 w^{h, i}$ & 3D T1w Post ${ }^{b}$ \\
\hline Sequence & $\mathrm{IR}_{-G R E^{\mathrm{e}, \mathrm{f}}}$ & $\mathrm{TSE}^{\mathrm{c}}$ & SS-EPI ${ }^{\mathrm{g}}$ & & $\mathrm{TSE}^{\mathrm{c}}$ & $\mathrm{IR}_{-G R E^{\mathrm{e}, \mathrm{f}}}$ \\
\hline Plane & Sagittal/Axial & Axial & Axial & & Axial & Sagittal/Axial \\
\hline Mode & $3 \mathrm{D}$ & $2 \mathrm{D}$ & $2 \mathrm{D}$ & & $2 \mathrm{D}$ & $3 \mathrm{D}$ \\
\hline $\mathrm{TR}[\mathrm{ms}]$ & $2100^{\mathrm{m}}$ & $>6000$ & $>5000$ & & $>2500$ & $2100^{\mathrm{m}}$ \\
\hline $\mathrm{TE}[\mathrm{ms}]$ & Min & $100-140$ & Min & & $80-120$ & Min \\
\hline $\mathrm{TI}[\mathrm{ms}]$ & $1100^{\mathrm{n}}$ & $2000-2500^{\mathrm{k}}$ & & & & $1100^{\mathrm{n}}$ \\
\hline Flip angle & $10^{\circ}-15^{\circ}$ & $90^{\circ} \geq 160^{\circ}$ & $90^{\circ} / 180^{\circ}$ & & $90^{\circ} \geq 160^{\circ}$ & $10^{\circ}-15^{\circ}$ \\
\hline Frequency & $\geq 172$ & $\geq 256$ & $\geq 128$ & & $\geq 256$ & $\geq 172$ \\
\hline Phase & $\geq 172$ & $\geq 256$ & $\geq 128$ & & $\geq 256$ & $\geq 172$ \\
\hline NEX & $\geq 1$ & $\geq 1$ & $\geq 1$ & & $\geq 1$ & $\geq 1$ \\
\hline FOV & $256 \mathrm{~mm}$ & $240 \mathrm{~mm}$ & $240 \mathrm{~mm}$ & & $240 \mathrm{~mm}$ & $256 \mathrm{~mm}$ \\
\hline Slice thickness & $\leq 1.5 \mathrm{~mm}$ & $\leq 4 \mathrm{~mm}^{1}$ & $\leq 4 \mathrm{~mm}^{1}$ & & $\leq 4 \mathrm{~mm}^{1}$ & $\leq 1.5 \mathrm{~mm}$ \\
\hline Gap/Spacing & 0 & 0 & $\overline{0}$ & & 0 & 0 \\
\hline Diffusion options ${ }^{p}$ & & & $\begin{aligned} b & =0,500,1000 \mathrm{~s} / \mathrm{mm}^{2} \\
& \geq 3 \text { directions }\end{aligned}$ & & & \\
\hline Parallel imaging & $\mathrm{Up}$ to $2 \mathrm{x}$ & Up to $2 \mathrm{x}$ & Up to $2 \mathrm{x}$ & & Up to $2 \mathrm{x}$ & $\mathrm{Up}$ to $2 \mathrm{x}$ \\
\hline $\begin{array}{l}\text { Approximate } \\
\text { scan time }\end{array}$ & $5-10 \mathrm{~min}$ & $4-8 \min$ & $2-4 \min$ & & $4-8 \min$ & $5-10 \mathrm{~min}$ \\
\hline
\end{tabular}

$\mathrm{Ax}=\mathrm{Axial} ; \mathrm{ADC}=$ apparent diffusion coefficient FLAIR $=$ fluid attenuated inversion recovery $; \mathrm{DWI}=$ diffusion-weighted imaging $3 \mathrm{D}=$ three dimensional; TSE = turbo spin echo; EPI = echo planar imaging; SS-EPI = single-shot echo planar imaging; GE-EPI = gradient echo echo planar imaging; $2 \mathrm{DFL}=$ two-dimensional FLASH (fast low angle shot) gradient recalled echo; MPRAGE $=$ magnetization prepared rapid gradient-echo; $\mathrm{A} / \mathrm{P}=\mathrm{anterior}$ to posterior; $\mathrm{R} / \mathrm{L}=$ right to left; $\mathrm{NEX}=$ number of excitations or averages; $\mathrm{FOV}=$ field of view; $\mathrm{TE}=$ echo time; $\mathrm{TR}=$ repetition time; $\mathrm{TI}=$ inversion time; $\mathrm{PD}=$ proton density; DSC = dynamic susceptibility contrast; IR-GRE = inversion-recovery gradient-recalled echo

${ }^{\mathrm{a}} 0.1 \mathrm{mmol} / \mathrm{kg}$ dose injection with a Gadolinium chelated contrast agent. Use of a power injector is desirable at an injection rate of 3-5cc/s

${ }^{\mathrm{b}}$ Post-contrast 3D T1-weighted images should be collected with equivalent parameters to pre-contrast 3D T1-weighted images

${ }^{\mathrm{c}}$ TSE $=$ turbo spin echo (Siemens \& Philips) is equivalent to FSE (fast spin echo; GE, Hitachi, Toshiba)

${ }^{\mathrm{d}}$ FL2D = two-dimensional fast low angle shot (FLASH; Siemens) is equivalent to the spoil gradient recalled echo (SPGR; GE) or T1- fast field echo (FFE; Philips), fast field echo (FastFE; Toshiba), or the radiofrequency spoiled steady state acquisition rewound gradient echo (RSSG; Hitachi). A fast gradient echo sequence without inversion preparation is desired

${ }^{\mathrm{e}}$ IR-GRE = inversion-recovery gradient-recalled echo sequence is equivalent to MPRAGE = magnetization prepared rapid gradient-echo (Siemens \& Hitachi) and the inversion recovery spoiled gradient-echo (IR-SPGR or Fast SPGR with inversion activated or BRAVO; GE), 3D turbo field echo (TFE; Philips), or 3D fast field echo (3D Fast FE; Toshiba)

${ }^{\mathrm{f}} \mathrm{A} 3 \mathrm{D}$ acquisition without inversion preparation will result in different contrast compared with MPRAGE or another IR-prepped 3D T1-weighted sequences and therefore should be avoided

${ }^{\mathrm{g}}$ In the event of significant patient motion, a radial acquisition scheme may be used (e.g. BLADE [Siemens], PROPELLER [GE], MultiVane [Philips], RADAR [Hitachi], or JET [Toshiba]); however, this acquisition scheme is can cause significant differences in ADC quantification and therefore should be used only if EPI is not an option. Further, this type of acquisition takes considerably more time

${ }^{\mathrm{h}}$ Dual echo PD/T2 TSE is optional for possible quantification of tissue T2. For this sequence, the PD echo is recommended to have a TE $<25 \mathrm{~ms}$

${ }^{\mathrm{i}}$ Advanced sequences can be substituted into this time slot, so long as 3D post-contrast T1-weighted images are collected between 4 and 8 min after contrast injection

${ }^{\mathrm{j}} 3 \mathrm{D}$ FLAIR is an optional alternative to 2D FLAIR, with sequence parameters as follows per EORTC guidelines: 3D TSE/FSE acquisition; TE $=90$ $140 \mathrm{~ms}$; TR $=6000-10000 \mathrm{~ms}$; TI $=2000-2500 \mathrm{~ms}$ (chosen based on vendor recommendations for optimized protocol and field strength); GRAPPA $\leq 2$; Fat Saturation; Slice thickness $\leq 1.5 \mathrm{~mm}$; Orientation Sagittal or Axial; FOV $\leq 250 \mathrm{~mm} \times 250 \mathrm{~mm}$; Matrix $\geq 244 \times 244$

${ }^{\mathrm{k}}$ Choice of TI should be chosen based on the magnetic field strength of the system (e.g. TI $\approx 2000 \mathrm{~ms}$ for $1.5 \mathrm{~T}$ and TI $\approx 2500 \mathrm{~ms}$ for $3 \mathrm{~T}$ )

${ }^{1}$ In order to ensure comparable SNR older 1.5T MR systems can use contiguous (no interslice gap) images with $5 \mathrm{~mm}$ slice thickness or increase NEX for slice thickness $\leq 4 \mathrm{~mm}$

${ }^{\mathrm{n}}$ For Siemens and Hitachi scanners. GE, Philips, and Toshiba scanners should use a TI $=400-450 \mathrm{~ms}$ for similar contrast

${ }^{\mathrm{m}}$ For Siemens and Hitachi scanners. GE, Philips, and Toshiba scanners should use a TR $=5-15 \mathrm{~ms}$ for similar contrast

${ }^{\mathrm{p}}$ Older model MR scanners that are not capable of $>2 b$-values should use $b=0$ and $1000 \mathrm{~s} / \mathrm{mm}^{2}$

products or calcifications [67, 68]. Further, the American College of Radiology (ACR) recommends this approach for identification and delineation of subtly enhancing bone and soft tissue lesions [69]. 
Fig. 1 Construction of contrast enhanced T1-weighted subtraction maps in a recurrent glioblastoma patient treated with bevacizumab. A) Pre-contrast T1weighted MR image. B) Postcontrast T1-weighted MR image. C) T1 subtraction map calculated by voxel-wise subtraction of precontrast from post-contrast T1weighted images highlighting areas of increased contrast enhancement. Red arrows show two subtly enhancing lesions that are easily identified on $\mathrm{T} 1$ subtraction maps
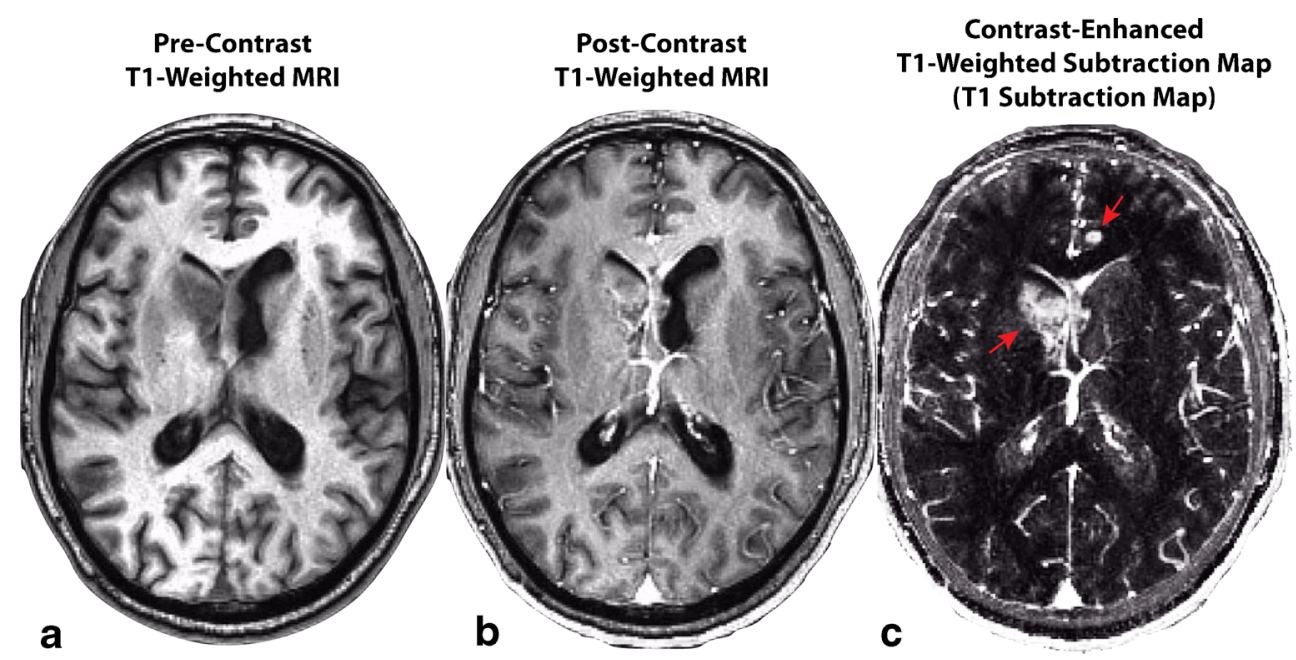

\section{Bidimensional and/or Volumetric Measurements}

Similar to the current RANO criteria, two-dimensional, perpendicular measurements of contrast enhancing tumor size, excluding the resection cavity along with any cysts or areas of central macroscopic necrosis, should be used for response assessment if volumetric tools are not available. Table 2 outlines suggested volumetric conversions from two- to threedimensional measurements for consistency in response definitions, as outlined by Chappell et al. [70].

It is important to note that the field remains conflicted on whether or not enhancing disease should be included in tumor size measurements, or whether it is more appropriate to monitor total enhancing lesion volume, which may include central macroscopic necrosis and any cystic components (but excluding surgically resected tissue). Scientific studies have shown that both approaches for quantifying change in tumor size as a surrogate of treatment response are valuable. Multiple studies utilizing the Macdonald and RANO criteria have shown that change in enhancing disease size using bidimensional measurements, excluding necrosis and cystic components, can be used to predict survival in a variety of therapies. A recent study from the BRAIN trial, a phase II trial of bevacizumab with or without irinotecan in recurrent GBM, confirmed that change in the volume of enhancing disease can be used to predict survival benefit [66]. However, a recent study examining growth rates in treatment naïve presurgical GBMs showed that changes in enhancing disease only may not be reliable, since changes occurring prior to any therapy often showed stable or decreasing tumor enhancing disease volume [61]. Growth rates were universally positive (i.e. growing) when total lesion volume (including central necrosis) were taken into consideration, which appears more realistic given the fast growth trajectory of these tumors during therapeutic intervention. Regardless, future studies are warranted to determine which measurement may be more clinically meaningful or reliable in predicting early response to new therapies.

\section{Definition of Measurable Disease, Non-Measurable Disease, and Target Lesions}

Measurable disease should be defined as contrast enhancing lesions with a minimum size of both perpendicular measurements greater than or equal to $10 \mathrm{~mm}$ (Fig. 2). For example, if the largest diameter is $15 \mathrm{~mm}$ but the perpendicular diameter is $8 \mathrm{~mm}$, this would constitute non-measurable disease. Additionally, in the event that the BTIP protocol is not used, if the slice thickness plus interslice gap is greater than $5 \mathrm{~mm}$, then the minimum size for both perpendicular measurements should be twice the sum of the slice thickness and interslice gap (e.g. if the slice thickness is $5 \mathrm{~mm}$ with $1.5 \mathrm{~mm}$ interslice gap, the minimum tumor size on both perpendicular dimensions should be $13 \mathrm{~mm}$ ). Up to a total of five target measurable lesions should be defined and ranked from largest to smallest (Fig. 2).
Table 2 Bidimensional to volumetric definitions $[54,70$, 96] of radiographic response and progression

\begin{tabular}{lll}
\hline State of disease & Change in bidimensional product & Estimated volumetric change \\
\hline Complete response (CR) & $100 \%$ Decrease & $100 \%$ Decrease \\
Partial response (PR) & $\geq 50 \%$ Decrease & $\geq 65 \%$ Decrease \\
Progressive disease (PD) & $\geq 25 \%$ Increase & $\geq 40 \%$ Increase \\
Stable disease (SD) & $<50 \%$ Decrease to & $<65 \%$ Decrease to \\
& $<25 \%$ Increase & $<40 \%$ Increase \\
\hline
\end{tabular}




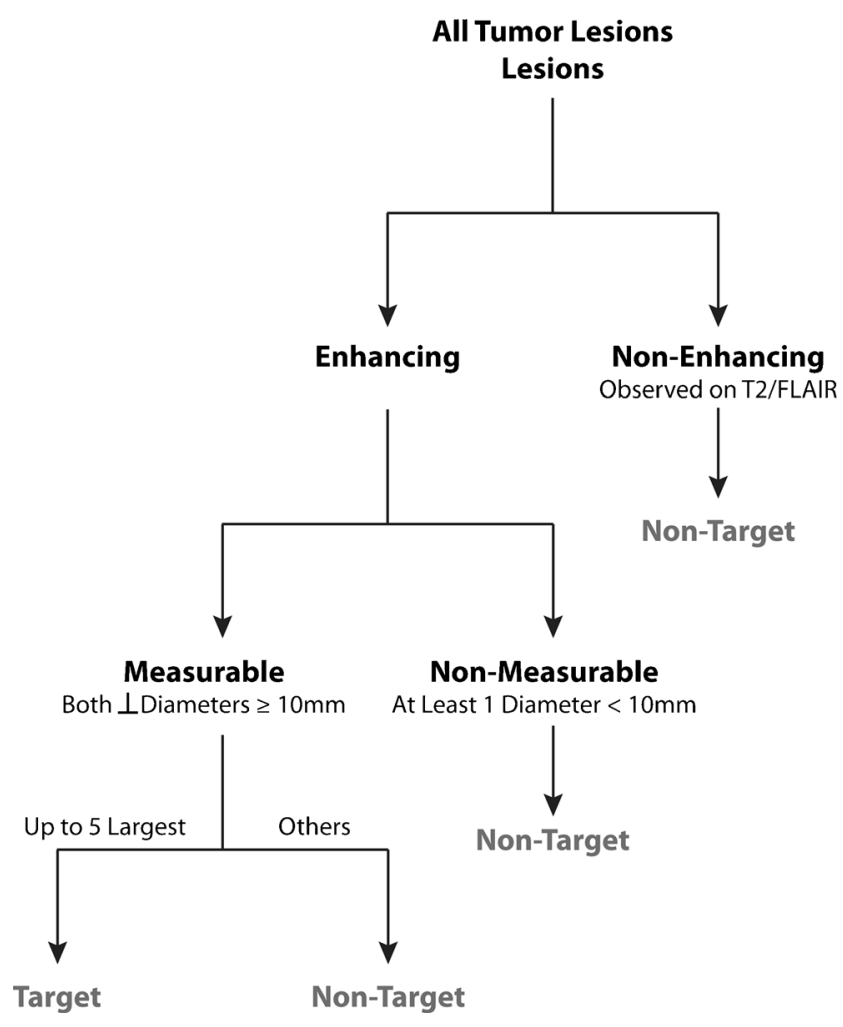

Fig. 2 Algorithm for identifying measurable and target lesions

Non-measurable disease should be defined as lesions that are too small to be measured (less than $1 \mathrm{~cm}$ in both perpendicular dimensions), lesions that lack contrast enhancement (non-enhancing disease), or lesions that contain a poorly defined margin that cannot be measured or segmented with confidence.

\section{Correction for "Baseline Tumor Volume" in Newly Diagnosed and Recurrent GBM}

An abundance of single center, multicenter, and phase I-III trials have confirmed that baseline contrast enhancing tumor size (volume or bidirectional measurements) is a significant prognostic factor contributing to overall survival (OS) in GBM. In newly diagnosed GBM, both extent of resection $[3,71-87]$ and post-surgical residual volume [83-85, 88-92] have been shown to be prognostic. Similarly, baseline pretreatment contrast enhanced tumor size has also been shown to be prognostic for OS in recurrent GBM [53, 66, 93]. However, from a clinical trial perspective, post-surgical residual enhancing tumor volume may be a more practical measurement to obtain, as pre-surgical MRI scans are often not available or collected as part of clinical trials because patients are not enrolled until after surgery and diagnosis. Thus, care should be made to make sure baseline tumor size is a stratification factor during randomization (i.e. prospectively balanced across treatment arms) and used as a covariate in statistical models evaluating treatment efficacy.

\section{Post-Radiation MRI Examination as the Reference for Evaluating Radiographic Response in Newly Diagnosed GBM}

The current RANO criterion defines the post-surgical MRI scan as the baseline for treatment response evaluation; however, we propose using the post-radiation examination (i.e. the first scan following completion of concurrent radiation therapy and chemotherapies such as temozolomide and/or experimental therapeutics) as the baseline for response assessment because reliability of tumor assessment on the post-surgical MR scans can be problematic for a number of reasons. First, this scan is typically acquired prior to a final pathological diagnosis, thus patients are not yet enrolled in a clinical trial and therefore the imaging protocol may not be consistent with trial recommendations, leading to a mismatch between the baseline and subsequent follow-up time points. Secondly, post-operative MR scans are often contaminated with post-surgical changes including blood products and increased vascular permeability from surgical trauma. Thirdly, steroid dose can be highly variable during this time and may be poorly annotated, as patients are typically not yet enrolled in clinical trials at this point. Additionally, the timing of the post-operative MR scans can be highly variable from patient to patient, depending on the complexity of the surgery and potential intraoperative complications, and institution by institution, as many factors including availability of inpatient MR scanners can lead to different timing of the post-surgical MRI evaluation. This variability inevitably leads to differing degrees of post-surgical artifacts and fluid levels on the resulting images. Together, these factors appear to indicate the post-surgical MRI examination may not be a reliable reference scan for accurately determining radiographic changes, despite post-surgical residual enhancing volume being a significant prognostic factor as outlined above.

Perhaps the most compelling argument for using the postradiation scan as the baseline for determining response assessment is the highly unpredictable, transient radiographic changes that often accompany the initial chemoradiation phase (i.e. external beam radiation therapy plus concurrent temozolomide) with or without experimental therapeutics. Within 1 month after completion of standard chemoradiation therapy, approximately $50 \%$ of patients will experience radiographic changes suggestive of early tumor progression in reference to the post-surgical MRI exam, of which $50 \%$ are likely to have pseudoprogression (i.e. $25 \%$ of all patients at 1 month post-chemoradiation are estimated to have pseudoprogression) [94]. This proportion of patients with both early progression and pseudoprogression decreases steadily during the subsequent standard adjuvant chemotherapy phase, which forms the basis for current RANO recommendations of excluding patients in recurrent GBM trials who progressed within 3 months after completion of chemoradiation. Many clinicians are reluctant to change therapy based on this examination due to the relatively high incidence of 
treatment-related radiographic changes directly after completion of concurrent chemotherapy and radiation, and instead use this scan as a new baseline in which to interpret subsequent changes in tumor size. Additionally, experimental therapeutics that significantly alter vascular permeability, including antiangiogenic and immunotherapies, when used concurrently with radiation therapy and temozolomide often demonstrate dramatic and transient changes in contrast enhancement that quickly stabilize following completion of radiation [95]. Despite the improved lesion conspicuity on $\mathrm{T} 1$ subtraction maps in the settings of these therapies, these early changes between the post-surgical, pre-radiation exam and the post-radiation exam may not accurately reflect true changes in tumor burden nor predict long-term survival benefit [95].

\section{Detailed Definitions Used for Modified Radiographic Response Assessment Criteria}

Radiographic response should be determined in comparison to the tumor measurements obtained at baseline (post-radiation scan will be baseline for newly diagnosed GBM and pretreatment scans will be the baseline for recurrent GBM) for determination of response, and the smallest tumor measurement at either pre-treatment baseline or following initiation of therapy for determining progression.

Because novel treatments are likely to result in a higher than normal incidence of treatment-related increase in contrast enhancement ("pseudoprogression", PsP) or decrease in contrast enhancement ("pseudoresponse", PsR), patients should continue therapy with close observation (e.g. 4-8 week intervals) if there is a suspicion of PsP or PsR. If subsequent imaging studies and/or clinical observations demonstrate that progression in fact has occurred, the date of confirmed progression should be noted as the scan at which the potential progression was first identified. Definitions for complete response, partial response, progressive disease, and stable disease should be defined as follows for all target lesions.

Complete Response $(C R)$ : Requires all of the following:

1. Disappearance of all enhancing measurable and nonmeasurable disease sustained for at least 4 weeks. The first scan exhibiting disappearance of all enhancing measurable and non-measurable disease is considered "preliminary CR". If the second scan exhibits measurable enhancing disease with respect to the "preliminary CR" scan, then the response is not sustained, noted as pseudoresponse, PsR, and is now considered "preliminary PD" (note confirmed PD requires at least two sequential increases in tumor volume). If the second scan continues to exhibit disappearance of enhancing disease or emergence of non-measurable disease (less than $10 \mathrm{~mm}$ bidimensional product), it is considered a durable $C R$ and the patient should continue on therapy until confirmed $\mathrm{PD}$ is observed.

2. Patients must be off corticosteroids (or on physiologic replacement doses only).

3. Stable or improved clinical assessments (i.e. neurological examinations).

Note: Patients with non-measurable disease only at baseline cannot have CR; the best response possible is stable disease (SD).

Partial Response (PR): Requires all of the following:

1. $\geq 50 \%$ decrease in sum of products of perpendicular diameters or $\geq 65 \%$ decrease in total volume $[54,70,96]$ of all measurable enhancing lesions compared with baseline, sustained for at least 4 weeks. The first scan exhibiting $\geq 50 \%$ decrease in sum of products of perpendicular diameters or $\geq 65 \%$ decrease in total volume $[54,70,96]$ of all measurable enhancing lesions compared with baseline is considered "preliminary PR". If the second scan exhibits PD with respect to the "preliminary PR" scan, then the response is not sustained, noted as pseudoresponse, PsR, and is now considered "preliminary PD" (note confirmed $\mathrm{PD}$ requires at least two sequential increases in tumor volume). If the second scan exhibits $\mathrm{SD}, \mathrm{PR}$, or $\mathrm{CR}$, it is considered a durable $P R$ and the patient should continue on therapy until confirmed PD is observed.

2. Steroid dose should be the same or lower compared with baseline scan.

3. Stable or improved clinical assessments.

Note: Patients with non-measurable disease only at baseline cannot have PR; the best response possible is stable disease (SD).

Progressive Disease (PD): Defined by any of the following:

1. At least two sequential scans separated by at $\geq 4$ weeks both exhibiting $\geq 25 \%$ increase in sum of products of perpendicular diameters or $\geq 40 \%$ increase in total volume $[54,70,96]$ of enhancing lesions. The first scan exhibiting $\geq 25 \%$ increase in sum of products of perpendicular diameters or $\geq 40 \%$ increase in total volume $[54,70,96]$ of enhancing lesions should be compared to the smallest tumor measurement obtained either at baseline (if no decrease) or best response (on stable or increasing steroid dose) and is noted as "preliminary PD." If the second scan at least 4 weeks later exhibits a subsequent $\geq 25 \%$ increase in sum of products of perpendicular diameters or $\geq 40 \%$ increase in total volume of enhancing lesions relative to the "preliminary PD" scan, it is considered "confirmed PD" and the patient should discontinue therapy. If the second scan at least 4 weeks later exhibits $\mathrm{SD}$ or PR/ $\mathrm{CR}$, this scan showing "preliminary PD" is noted as 
"pseudoprogression", PsP, and the patient should continue on therapy until a second increase in tumor size relative to the PsP scan is observed. Note that any new measurable ( $>10 \mathrm{~mm} \times 10 \mathrm{~mm}$ ) enhancing lesions should not be immediately considered PD, but instead should be added to the sum of bidimensional products or total volume representing the entire enhancing tumor burden.

2 . In the case where the baseline or best response demonstrates no measurable enhancing disease (visible or not visible), then any new measurable ( $>10 \mathrm{~mm} \times 10 \mathrm{~mm})$ enhancing lesions are considered PD after confirmed by a subsequent scan $\geq 4$ weeks exhibiting $\geq 25 \%$ increase in sum of products of perpendicular diameters or $\geq 40 \%$ increase in total volume of enhancing lesions [54, 70, 96] relative to the scan first illustrating new measurable disease. The first scan exhibiting new measurable disease is noted as "preliminary PD." If the second scan at least 4 weeks later exhibits a subsequent $\geq 25 \%$ increase in sum of products of perpendicular diameters or $\geq 40 \%$ increase in total volume $[54,70,96]$ of enhancing lesions relative to the "preliminary PD" scan it is considered "confirmed PD" and the patient should discontinue therapy. If the second scan at least 4 weeks later exhibits $\mathrm{SD}, \mathrm{CR}, \mathrm{PR}$, or becomes non-measurable, this scan showing "preliminary PD" is noted as "pseudoprogression", PsP, and the patient should continue on therapy until a second increase in tumor size relative to the "preliminary PD", or PsP, scan is observed. Note that any new measurable ( $>10 \mathrm{~mm} \times 10 \mathrm{~mm}$ ) enhancing lesions on the subsequent scan following the preliminary PD scan should not be immediately considered confirmed PD, but instead should be added to the sum of bidimensional products or total volume representing the entire enhancing tumor burden.

3. Clear clinical deterioration not attributable to other causes apart from tumor (e.g. seizures, medication adverse effects, therapy complications, stroke, infection) or attributable to changes in steroid dose.

4. Failure to return for evaluation as a result of death or deteriorating condition.

Stable Disease $(S D)$ : Requires all of the following:

1. Does not qualify for CR, PR, or PD as defined above. Note this also applies to patients that demonstrate PsR when the confirmation scan does not show PD or PsP when the confirmation scan does not show PR/CR.

2. In the event that corticosteroid dose was increased (for new symptoms/signs) without confirmation of disease progression on neuroimaging, and subsequent follow-up imaging shows that the steroid increase was required because of disease progression, the last scan considered to show stable disease will be the scan obtained when the corticosteroid dose was equivalent to the baseline dose.

\section{Symptomatic Deterioration \& Reporting Clinical Status}

Patients with global deterioration of health status requiring discontinuation of treatment without objective evidence of disease progression at that time, and not either related to study treatment or other medical conditions, should be reported as PD due to "symptomatic deterioration." Every effort should be made to document the objective progression even after discontinuation of treatment due to symptomatic deterioration. Neurological exam data should be provided to the independent radiologic facility as "stable, better, worse" in case report forms or from study sponsor. Clinical status should be recorded as "worse" if the neurological exam is worse, otherwise the clinical status should be set to "not worse." In the event that necessary clinical data is not available, clinical status should be recorded as "not available" and that particular time point can only be reviewed for PD (otherwise "non-evaluable"). Neurological data must be within \pm 7 days of the time-point response date, otherwise the data is considered "not available".

\section{Steroid Use and Dose}

Steroid use should be derived from the concomitant medications on the case report forms and recorded as "Yes", "No", or "not available". A value of "No" should be assigned if, at the timepoint, the subject is not on steroids or on physiologic replacement doses only ( $<1.5 \mathrm{mg}$ dexamethasone or equivalent per day).

Steroid dose should be derived from the concomitant medications on the case report forms. Average steroid dose no greater than $2 \mathrm{mg}$ change from baseline should be abstracted to "stable". If outside this range the steroid dose should be abstracted to "increased" or "decreased" accordingly. Steroid data should be within \pm 5 days of the time-point response date, otherwise the data is considered "not available".

\section{Overall Objective Status}

The overall objective status for an evaluation should be determined by combining the patient's radiographic response on target lesions, new disease, neurological status, and steroid dose/ usage as defined in Table 3 for patients with measurable ( $>10 \mathrm{~mm} \times 10 \mathrm{~mm}$ ) disease. Note that patients with possible PsP or pseudoresponse should be given the Objective Status of "Preliminary Progression" or "Preliminary Response", respectively. Once PsP, pseudoresponse, or true progression/response are confirmed, the Objective Status can be changed accordingly.

\section{Detailed Modified Radiographic Response Assessment Rubric}

In order to provide both clinical guidelines for continuing therapy beyond suspected radiographic progression if the 
Table 3 Guidelines for determining comprehensive objective status

\begin{tabular}{|c|c|c|c|c|c|c|}
\hline $\begin{array}{l}\text { Target lesions } \\
\text { (current scan) }\end{array}$ & $\begin{array}{l}\text { Target lesions (previous } \\
\text { scan) }\end{array}$ & $\begin{array}{l}\text { New sites of } \\
\text { measurable } \\
\text { disease }^{a}\end{array}$ & $\begin{array}{l}\text { Neurological } \\
\text { status }\end{array}$ & $\begin{array}{l}\text { Steroid } \\
\text { usage }\end{array}$ & Steroid dose & $\begin{array}{l}\text { Overall objective } \\
\text { status }\end{array}$ \\
\hline $\mathrm{CR}$ & Not Evaluated & No & Stable/Better & No & N/A & Preliminary CR \\
\hline PR & Not Evaluated & No & Stable/Better & Any & Stable/Decreasing & Preliminary PR \\
\hline PD & Not Evaluated & Yes or No & Stable/Better & Any & Stable/Increasing & Preliminary PD \\
\hline PD & Preliminary or Confirmed PR/CR & No & Stable/Better & Any & Stable/Increasing & Preliminary PD \\
\hline SD & $\begin{array}{l}\text { Preliminary or Confirmed } \\
\mathrm{CR} / \mathrm{PR} \text { or } \mathrm{SD} / \mathrm{NE}\end{array}$ & No & Stable/Better & Any & N/A & SD \\
\hline PR & Preliminary PR & Yes or No & Stable/Better & Any & Stable/Decreasing & Confirmed PR \\
\hline SD & Preliminary PR & Yes or No & Stable/Better & Any & Stable/Decreasing & $\begin{array}{l}\text { SD (Preliminary PR } \\
\rightarrow \text { Confirmed PR) }\end{array}$ \\
\hline SD & Preliminary CR & Yes or No & Stable/Better & Any & Stable/Decreasing & $\begin{array}{r}\text { SD (Preliminary CR } \\
\rightarrow \text { Confirmed CR) }\end{array}$ \\
\hline $\mathrm{CR}$ & Preliminary CR & No & Stable/Better & No & N/A & Confirmed CR \\
\hline SD & Preliminary PD & No & Stable/Better & Any & Stable/Decreasing & $\begin{array}{l}\text { SD } \\
\text { (Confirmed PsP) }\end{array}$ \\
\hline $\begin{array}{l}\mathrm{CR} / \mathrm{PR} / \mathrm{SD} \\
\mathrm{PD} / \mathrm{NE}\end{array}$ & $\mathrm{CR} / \mathrm{PR} / \mathrm{SD} / \mathrm{PD} / \mathrm{NE}$ & Yes or No & Worse & Any & Stable/Increasing & Confirmed PD \\
\hline PD & Preliminary PD & Yes or No & Any & Yes & Stable/Increasing & Confirmed PD \\
\hline
\end{tabular}

${ }^{a}$ Note that new sites of measurable disease are added to the sum of bidimensional products or total lesion volume, or constitutes preliminary PD in the case of no measurable disease at baseline or best response

treating physician believes there may be a therapeutic benefit and to provide criteria for defining progression and early drug failure while also allowing for the possibility of PsP and PsR, a modified response rubric similar to those described recently [97] should be employed. Two different rubrics should be used depending on whether the patient is newly diagnosed or enrolled in a trial for recurrent disease.

It is important to note that the primary differences between conventional RANO and the proposed modified criteria are: (1) use of the post-radiation time point as the baseline for response evaluation in newly diagnosed GBM and (2) considering only objectively defined, measurable enhancing disease in the definition of response and progression (i.e. exclusion of qualitatively assessed T2/FLAIR changes).

\section{Newly Diagnosed GBM (Fig. 3)}

Newly diagnosed GBM patients will initially undergo a preentry MRI scan for initial diagnosis prior to entry in the study and prior to therapy. The post-operative scan $[\mathrm{MRI}(0)]$ is

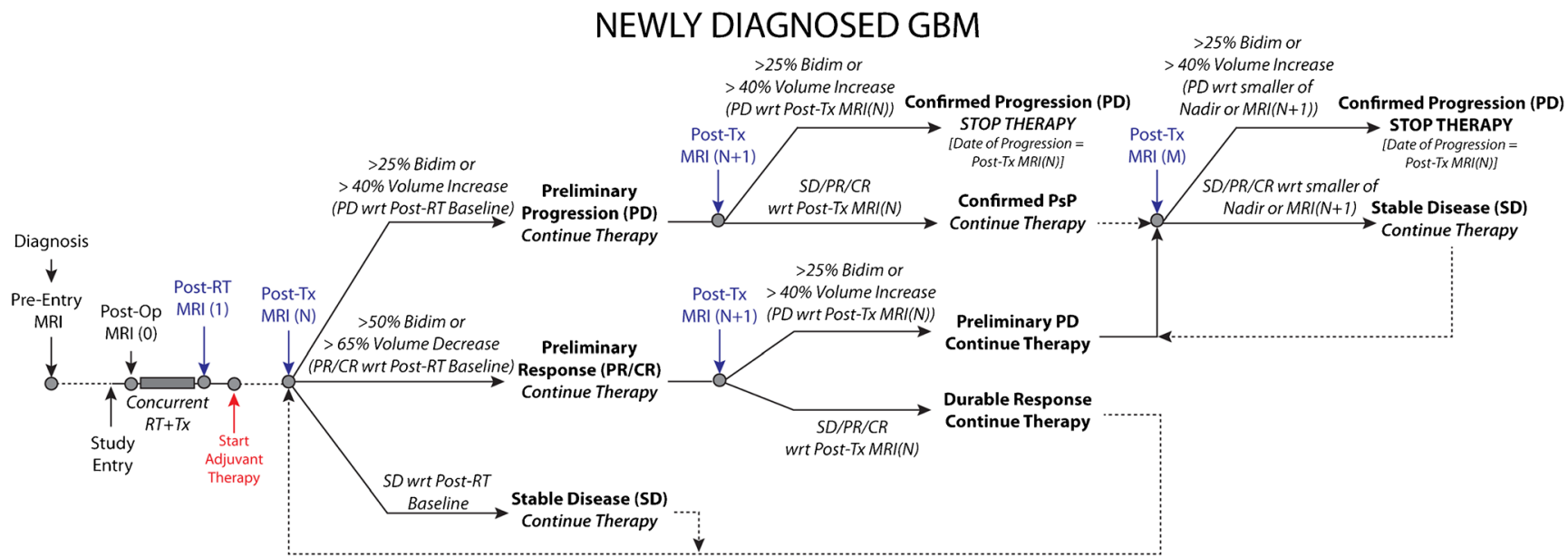

Fig. 3 Modified radiographic response assessment rubric for management of both pseudoprogression and pseudoresponse in newly diagnosed glioblastoma 


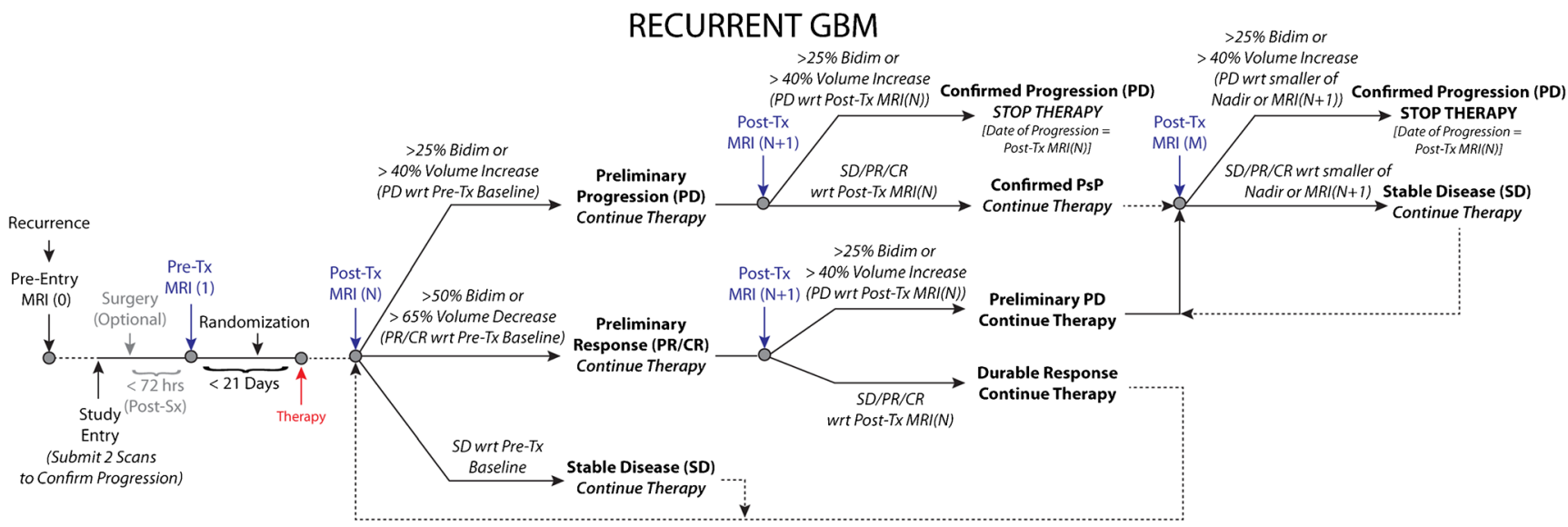

Fig. 4 Modified radiographic response assessment rubric for recurrent glioblastoma

desired in order to assess residual enhancing disease volume for use as a covariate in survival analyses, as described previously. Patients will then start on standard or experimental therapy with concurrent radiation therapy (RT). The Post-RT scan $[M R I(1)]$ will be required and used as the baseline scan for which response will be determined. ${ }^{1}$ Following the first cycles of adjuvant therapy, patients will receive additional required $\mathrm{MRI}$ scans [MRI(N)].

\section{Recurrent GBM (Fig. 4)}

Recurrent GBM patients will undergo a pre-entry MRI scan $[\mathrm{MRI}(0)]$ at the time of recurrence. At the time of study entry, two scans to confirm progression should be submitted consisting of at least one scan at the time of progression and one scan at Nadir or baseline. If the patient undergoes surgery (optional), then the post-surgical, pre-treatment MRI can be used as the baseline [MRI(1)], assuming it is obtained $<72$ hours from surgery to reduce post-operative reactive enhancement $[91,98]$. (Note: If the post-operative MRI scan is used as the baseline reference, the standardized MRI protocols must be used.) If the patient does not go to surgery or if the start of treatment is $>21$ days from the start of therapy, the patient will undergo a pre-treatment MRI [MRI(1)] scan as the baseline scan for which response will be determined. Following the first cycles of therapy, patients will receive additional MRI scans [MRI(N)].

\footnotetext{
${ }^{1}$ Note the post-surgical, pre-RT scan is often corrupted with post-surgical blood products and other surgical changes. Further, it is difficult to standardize in terms of post-surgical timing, where delays in scanning often lead to increased $\mathrm{T} 1$ shortening on pre-contrast T1-weighted images due to blood products, etc., and therefore may not be an adequate representation of baseline tumor burden.
}

\section{Details Common to Both Newly Diagnosed and Recurrent GBM}

Preliminary Radiographic Progression If the lesion size has increased $\geq 25 \%$ sum of bidirectional product or $\geq 40 \%$ in volume between MRI Scan 1 and N, these patients should be categorized as "preliminary radiographic progression". If the investigator believes the patient can safely continue on therapy, then they should continue to treat and acquire a follow-up confirmatory scan $[\mathrm{MRI}(\mathrm{N}+1)]$ at the next scan interval $(8$ weeks \pm 4 weeks from MRI Scan (N) or no less than 4 weeks minimum duration between preliminary PD and confirmed PD scans) to verify tumor growth and progression. For patients with gross-total resection (GTR) and no measurable enhancing disease, preliminary radiographic progression is defined as a transition from no measurable disease to nonmeasureable (but present) disease $(<10 \mathrm{~mm} \times 10 \mathrm{~mm})$ or measurable disease $(>10 \mathrm{~mm} \times 10 \mathrm{~mm})$. If the investigator feels it is safe to keep the patient on, a confirmatory scan at MRI $(\mathrm{N}+1)$ should be obtained to verify tumor progression.

Confirmed Progression If the patient has an increase $\geq 25 \%$ sum of bidirectional product or $\geq 40 \%$ in volume between MRI Scan $\mathrm{N}$ and $\mathrm{N}+1$, this is "Confirmed Progression", the patient should stop therapy and the date of radiographic progression is the date of suspected progression, MRI(N). If the patient has $\mathrm{SD} / \mathrm{PR} / \mathrm{CR}$ on MRI(N+1) with respect to MRI(N), PsP is confirmed and the patient should continue on therapy. Patients will then continue on therapy and receive additional follow-up MRI scans [MRI(M)]. If the lesion size has increased $\geq 25 \%$ sum of bidirectional product or $\geq 40 \%$ in volume on MRI(M) relative to the smaller of Nadir or MRI(N + 1), then the patient has "Confirmed Progression", the patient should stop therapy and the date of radiographic progression is the new date, MRI(M). For patients with no measurable disease at the Post-RT baseline, "Confirmed Progression" will be defined as a transition from non-measurable (but present) 
disease $(<10 \mathrm{~mm} x<10 \mathrm{~mm})$ on MRI(N) to measurable disease $(>10 \mathrm{~mm} \times 10 \mathrm{~mm})$ on MRI $(\mathrm{N}+1)$. For patients with confirmed $\mathrm{PsP}$ and no measurable disease at Nadir, "Confirmed Progression" should be defined as a transition from no measurable disease to measurable disease (>10mm $\times 10 \mathrm{~mm})$. In all cases, patients with confirmed progression should stop therapy.

Preliminary \& Confirmed Radiographic Response If a measurable lesion has decreased $\geq 50 \%$ sum of bidirectional product or $\geq 65 \%$ in volume between MRI(1) and MRI(N), these patients should be categorized as "preliminary radiographic responders" and will be monitored for an additional time point and/or treatment cycle. After an additional cycle of therapy ( 8 weeks \pm 4 weeks from $\mathrm{MRI}(\mathrm{N})$ ), patients will receive a confirmatory $\mathrm{MRI}(\mathrm{N}+1)$. If the lesion(s) have increased $\geq 25 \%$ sum of bidirectional product or $\geq 40 \%$ in volume from MRI(N) (indicating radiographic progression from $\operatorname{MRI}(\mathrm{N})$ ), this is considered an "unsustained radiographic response" or "pseudoresponse". The date of radiographic progression for these patients will be MRI(N+1) and the patient should stop therapy. Alternatively, if the lesion has not increased from MRI(N), this is considered a "durable radiographic response," the patient will continue on therapy, and the date of preliminary radiographic progression is the time point of an increase $\geq 25 \%$ sum of bidirectional product or $\geq 40 \%$ in volume (from Nadir) during the remainder of the study. The investigator can then decide whether to continue safely on therapy until progression has been confirmed and at the subsequent time point stop therapy if they feel the patient cannot safely continue therapy.

Stable Disease If the lesion size has not increased or decreased beyond the set thresholds between Scan 1 and $\mathrm{N}$, the patient is considered "stable." Such patients will continue on therapy, and the date of preliminary progression is the time point of an increase $\geq 25 \%$ sum of bidirectional product or $\geq 40 \%$ in volume (from Nadir) during the remainder of the study. Upon preliminary progression the investigator can choose to either continue therapy and confirm progression or discontinue therapy. For cases with significant neurologic decline at the time of imaging progression as determined from $\operatorname{MRI}(\mathrm{N})$, a confirmatory scan at time point MRI $(\mathrm{N}+1)$ may not be possible or necessary. For these cases, it is appropriate to define $\mathrm{MRI}(\mathrm{N})$ as the progression time point.

\section{Conclusions}

Although radiographic response assessment is imperfect and many nuances exist, changes in contrast enhancing tumor are both clinically meaningful and appropriate for evaluating efficacy of new treatments in GBM. The outlined modifications in this report are meant to both build on the strengths of the current RANO criteria while providing potential solutions for many of the common challenges.

\section{Compliance with Ethical Standards}

Funding American Cancer Society (ACS) Research Scholar Grant (RSG-15-003-01-CCE) (Ellingson); National Brain Tumor Society (NBTS) Research Grant (Ellingson, Cloughesy); Art of the Brain (Cloughesy); Ziering Family Foundation in memory of Sigi Ziering (Cloughesy); Singleton Family Foundation (Cloughesy).

Open Access This article is distributed under the terms of the Creative Commons Attribution 4.0 International License (http:// creativecommons.org/licenses/by/4.0/), which permits unrestricted use, distribution, and reproduction in any medium, provided you give appropriate credit to the original author(s) and the source, provide a link to the Creative Commons license, and indicate if changes were made.

\section{References}

1. Ostrom QT, Gittleman H, Fulop J et al. CBTRUS statistical report: primary brain and central nervous system tumors diagnosed in the United States in 2008-2012. Neuro Oncol, 17 Suppl 4, iv1-iv62 (2015).

2. Stupp R, Mason WP, van den Bent MJ et al. Radiotherapy plus concomitant and adjuvant temozolomide for glioblastoma. $N$ Engl $J$ Med, 352(10), 987-996 (2005).

3. Stupp R, Hegi ME, Mason WP et al. Effects of radiotherapy with concomitant and adjuvant temozolomide versus radiotherapy alone on survival in glioblastoma in a randomised phase III study: 5-year analysis of the EORTC-NCIC trial. Lancet Oncol, 10(5), 459-466 (2009).

4. Weller M, Cloughesy T, Perry JR, Wick W. Standards of care for treatment of recurrent glioblastoma-are we there yet? Neuro Oncol, 15(1), 4-27 (2013).

5. Chinot OL, Wick W, Mason W et al. Bevacizumab plus radiotherapy-temozolomide for newly diagnosed glioblastoma. $N$ Engl J Med, 370(8), 709-722 (2014).

6. Gilbert MR, Dignam JJ, Armstrong TS et al. A randomized trial of bevacizumab for newly diagnosed glioblastoma. $N$ Engl J Med, 370(8), 699-708 (2014).

7. Lamborn KR, Yung WK, Chang SM et al. Progression-free survival: an important end point in evaluating therapy for recurrent highgrade gliomas. Neuro Oncol, 10(2), 162-170 (2008).

8. Provenzale JM, Ison C, Delong D. Bidimensional measurements in brain tumors: assessment of interobserver variability. AJR Am J Roentgenol, 193(6), W515-522 (2009).

9. Russell SM, Elliott R, Forshaw D, Golfinos JG, Nelson PK, Kelly PJ. Glioma vascularity correlates with reduced patient survival and increased malignancy. Surg Neurol, 72(3), 242-246; discussion 246-247 (2009)

10. Leon SP, Folkerth RD, Black PM. Microvessel density is a prognostic indicator for patients with astroglial brain tumors. Cancer, 77(2), 362-372 (1996).

11. Wesseling P, van der Laak JA, Link M, Teepen HL, Ruiter DJ. Quantitative analysis of microvascular changes in diffuse astrocytic neoplasms with increasing grade of malignancy. Hum Pathol, 29(4), 352-358 (1998). 
12. Yuan F, Salehi HA, Boucher Y, Vasthare US, Tuma RF, Jain RK. Vascular permeability and microcirculation of gliomas and mammary carcinomas transplanted in rat and mouse cranial windows. Cancer Res, 54(17), 4564-4568 (1994).

13. Hobbs SK, Monsky WL, Yuan F et al. Regulation of transport pathways in tumor vessels: role of tumor type and microenvironment. Proc Natl Acad Sci USA, 95(8), 4607-4612 (1998).

14. Monsky WL, Fukumura D, Gohongi $\mathrm{T}$ et al. Augmentation of transvascular transport of macromolecules and nanoparticles in tumors using vascular endothelial growth factor. Cancer Research, 59(16), 4129-4135 (1999).

15. Folkman J. Role of angiogenesis in tumor growth and metastasis. Semin Oncol, 29(6 Suppl 16), 15-18 (2002).

16. Kelly PJ, Daumas-Duport C, Scheithauer BW, Kall BA, Kispert DB. Stereotactic histologic correlations of computed tomographyand magnetic resonance imaging-defined abnormalities in patients with glial neoplasms. Mayo Clin Proc, 62(6), 450-459 (1987).

17. Kelly PJ, Daumas-Duport C, Kispert DB, Kall BA, Scheithauer BW, Illig JJ. Imaging-based stereotaxic serial biopsies in untreated intracranial glial neoplasms. J Neurosurg, 66(6), 865-874 (1987).

18. Macdonald DR, Cascino TL, Schold SC, Jr., Cairncross JG. Response criteria for phase II studies of supratentorial malignant glioma. J Clin Oncol, 8(7), 1277-1280 (1990).

19. Levin VA, Crafts DC, Norman DM, Hoffer PB, Spire JP, Wilson CB. Criteria for evaluating patients undergoing chemotherapy for malignant brain tumors. J Neurosurg, 47(3), 329-335 (1977).

20. Miller AB, Hoogstraten B, Staquet M, Winkler A. Reporting results of cancer treatment. Cancer, 47(1), 207-214 (1981).

21. Therasse P, Arbuck SG, Eisenhauer EA et al. New guidelines to evaluate the response to treatment in solid tumors. European Organization for Research and Treatment of Cancer, National Cancer Institute of the United States, National Cancer Institute of Canada. J Natl Cancer Inst, 92(3), 205-216 (2000).

22. Pope WB, Lai A, Nghiemphu P, Mischel P, Cloughesy TF. MRI in patients with high-grade gliomas treated with bevacizumab and chemotherapy. Neurology, 66(8), 1258-1260 (2006).

23. Chamberlain MC. MRI in patients with high-grade gliomas treated with bevacizumab and chemotherapy. Neurology, 67(11), 2089; author reply 2089 (2006).

24. Sathornsumetee S, Cao Y, Marcello JE et al. Tumor angiogenic and hypoxic profiles predict radiographic response and survival in malignant astrocytoma patients treated with bevacizumab and irinotecan. J Clin Oncol, 26(2), 271-278 (2008).

25. Norden AD, Young GS, Setayesh $\mathrm{K}$ et al. Bevacizumab for recurrent malignant gliomas: efficacy, toxicity, and patterns of recurrence. Neurology, 70(10), 779-787 (2008).

26. Bokstein F, Shpigel S, Blumenthal DT. Treatment with bevacizumab and irinotecan for recurrent high-grade glial tumors. Cancer, 112(10), 2267-2273 (2008).

27. Ananthnarayan S, Bahng J, Roring J et al. Time course of imaging changes of GBM during extended bevacizumab treatment. $J$ Neurooncol, 88(3), 339-347 (2008).

28. Kang TY, Jin T, Elinzano H, Peereboom D. Irinotecan and bevacizumab in progressive primary brain tumors, an evaluation of efficacy and safety. J Neurooncol, 89(1), 113-118 (2008).

29. de Groot JF, Yung WK. Bevacizumab and irinotecan in the treatment of recurrent malignant gliomas. Cancer J, 14(5), 279-285 (2008).

30. Zuniga RM, Torcuator R, Jain R et al. Efficacy, safety and patterns of response and recurrence in patients with recurrent high-grade gliomas treated with bevacizumab plus irinotecan. $J$ Neurooncol, 91(3), 329-336 (2009).

31. Batchelor TT, Sorensen AG, di Tomaso E et al. AZD2171, a panVEGF receptor tyrosine kinase inhibitor, normalizes tumor vasculature and alleviates edema in glioblastoma patients. Cancer Cell, 11(1), 83-95 (2007).
32. Friedman HS, Prados MD, Wen PY et al. Bevacizumab alone and in combination with irinotecan in recurrent glioblastoma. $J$ Clin Oncol, 27(28), 4733-4740 (2009).

33. Vredenburgh JJ, Desjardins A, Herndon JE, 2nd et al. Phase II trial of bevacizumab and irinotecan in recurrent malignant glioma. Clin Cancer Res, 13(4), 1253-1259 (2007).

34. Vredenburgh JJ, Desjardins A, Herndon JE, 2nd et al. Bevacizumab plus irinotecan in recurrent glioblastoma multiforme. J Clin Oncol, 25(30), 4722-4729 (2007).

35. Friedman HS, Petros WP, Friedman AH et al. Irinotecan therapy in adults with recurrent or progressive malignant glioma. J Clin Oncol, 17(5), 1516-1525 (1999).

36. Cloughesy TF, Filka E, Kuhn J et al. Two studies evaluating irinotecan treatment for recurrent malignant glioma using an every-3-week regimen. Cancer, 97(9 Suppl), 2381-2386 (2003).

37. Raymond E, Fabbro M, Boige V et al. Multicentre phase II study and pharmacokinetic analysis of irinotecan in chemotherapy-naive patients with glioblastoma. Ann Oncol, 14(4), 603-614 (2003).

38. Prados MD, Lamborn K, Yung WK et al. A phase 2 trial of irinotecan (CPT-11) in patients with recurrent malignant glioma: a North American Brain Tumor Consortium study. Neuro Oncol, 8(2), 189-193 (2006).

39. Hygino da Cruz LC, Jr., Rodriguez I, Domingues RC, Gasparetto EL, Sorensen AG. Pseudoprogression and pseudoresponse: imaging challenges in the assessment of posttreatment glioma. AJNR Am J Neuroradiol, 32(11), 1978-1985 (2011).

40. Gallego Perez-Larraya J, Lahutte M, Petrirena G et al. Response assessment in recurrent glioblastoma treated with irinotecanbevacizumab: comparative analysis of the Macdonald, RECIST, RANO, and RECIST + F criteria. Neuro Oncol, 14(5), 667-673 (2012).

41. Nowosielski M, Wiestler B, Goebel G et al. Progression types after antiangiogenic therapy are related to outcome in recurrent glioblastoma. Neurology, 82(19), 1684-1692 (2014).

42. Chamberlain MC. Radiographic patterns of relapse in glioblastoma. J Neurooncol, 101(2), 319-323 (2011).

43. Jain RK, di Tomaso E, Duda DG, Loeffler JS, Sorensen AG, Batchelor TT. Angiogenesis in brain tumours. Nat Rev Neurosci, 8(8), 610-622 (2007).

44. Ellingson BM, Lai A, Harris RJ et al. Probabilistic radiographic atlas of glioblastoma phenotypes. AJNR Am J Neuroradiol, 34(3), 533-540 (2013)

45. Naeini KM, Pope WB, Cloughesy TF et al. Identifying the mesenchymal molecular subtype of glioblastoma using quantitative volumetric analysis of anatomic magnetic resonance images. Neuro Oncol, 15(5), 626-634 (2013).

46. Carrillo JA, Lai A, Nghiemphu PL et al. Relationship between tumor enhancement, edema, IDH1 mutational status, MGMT promoter methylation, and survival in glioblastoma. AJNR Am J Neuroradiol, 33(7), 1349-1355 (2012).

47. Zinn PO, Mahajan B, Sathyan P et al. Radiogenomic mapping of edema/cellular invasion MRI-phenotypes in glioblastoma multiforme. PLoS One, 6(10), e25451 (2011).

48. Pope WB, Chen JH, Dong J et al. Relationship between gene expression and enhancement in glioblastoma multiforme: exploratory DNA microarray analysis. Radiology, 249(1), 268-277 (2008).

49. Wen PY, Macdonald DR, Reardon DA et al. Updated response assessment criteria for high-grade gliomas: response assessment in neurooncology working group. J Clin Oncol, 28(11), 1963-1972 (2010).

50. Henson JW, Ulmer S, Harris GJ. Brain tumor imaging in clinical trials. AJNR Am J Neuroradiol, 29(3), 419-424 (2008).

51. Sorensen AG, Batchelor TT, Wen PY, Zhang WT, Jain RK. Response criteria for glioma. Nat Clin Pract Oncol, 5(11), 634-644 (2008).

52. van den Bent MJ, Vogelbaum MA, Wen PY, Macdonald DR, Chang SM. End point assessment in gliomas: novel treatments limit usefulness of classical Macdonald's criteria. J Clin Oncol, 27(18), 2905-2908 (2009). 
53. Dempsey MF, Condon BR, Hadley DM. Measurement of tumor "size" in recurrent malignant glioma: 1D, 2D, or 3D? AJNR Am J Neuroradiol, 26(4), 770-776 (2005).

54. Shah GD, Kesari S, Xu R et al. Comparison of linear and volumetric criteria in assessing tumor response in adult high-grade gliomas. Neuro Oncol, 8(1), 38-46 (2006).

55. Hopper KD, Kasales CJ, Van Slyke MA, Schwartz TA, TenHave TR, Jozefiak JA. Analysis of interobserver and intraobserver variability in CT tumor measurements. AJR Am J Roentgenol, 167(4), 851-854 (1996).

56. Lavin PT, Flowerdew G. Studies in variation associated with the measurement of solid tumors. Cancer, 46(5), 1286-1290 (1980).

57. Quoix E, Wolkove N, Hanley J, Kreisman H. Problems in radiographic estimation of response to chemotherapy and radiotherapy in small cell lung cancer. Cancer, 62(3), 489-493 (1988).

58. Thiesse P, Ollivier L, Di Stefano-Louineau D et al. Response rate accuracy in oncology trials: reasons for interobserver variability. Groupe Francais d'Immunotherapie of the Federation Nationale des Centres de Lutte Contre le Cancer. J Clin Oncol, 15(12), 3507-3514 (1997).

59. Warr D, McKinney S, Tannock I. Influence of measurement error on assessment of response to anticancer chemotherapy: proposal for new criteria of tumor response. J Clin Oncol, 2(9), 1040-1046 (1984).

60. Galanis E, Buckner JC, Maurer MJ et al. Validation of neuroradiologic response assessment in gliomas: measurement by RECIST, two-dimensional, computer-assisted tumor area, and computer-assisted tumor volume methods. Neuro Oncol, 8(2), 156-165 (2006).

61. Ellingson BM, Nguyen HN, Lai A et al. Contrast-enhancing tumor growth dynamics of preoperative, treatment-naive human glioblastoma. Cancer, 122(11), 1718-1727 (2016).

62. Huang RY, Rahman R, Ballman KV et al. The impact of T2/FLAIR evaluation per RANO criteria on response assessment of recurrent glioblastoma patients treated with bevacizumab. Clin Cancer Res, 22(3), 575-581 (2016).

63. Boxerman JL, Zhang Z, Safriel Y et al. Early post-bevacizumab progression on contrast-enhanced MRI as a prognostic marker for overall survival in recurrent glioblastoma: results from the ACRIN 6677/ RTOG 0625 Central Reader Study. Neuro Oncol, 15(7), 945-954 (2013).

64. Wen PY, Cloughesy TF, Ellingson BM et al. Report of the jumpstarting brain tumor drug development coalition and FDA clinical trials neuroimaging endpoint workshop (January 30, 2014, Bethesda MD). Neuro Oncol, 16 Suppl 7, vii36-47 (2014).

65. Ellingson BM, Bendszus M, Boxerman J et al. Consensus recommendations for a standardized brain tumor imaging protocol in clinical trials. Neuro Oncol, 17(9), 1188-1198 (2015).

66. Ellingson BM, Kim HJ, Woodworth DC et al. Recurrent glioblastoma treated with bevacizumab: contrast-enhanced T1-weighted subtraction maps improve tumor delineation and aid prediction of survival in a multicenter clinical trial. Radiology, 271(1), 200-210 (2014).

67. Melhem ER, Mehta NR. Dynamic T1-weighted spin-echo MR imaging: the role of digital subtraction in the demonstration of enhancing brain lesions. J Magn Reson Imaging, 9(4), 503-508 (1999).

68. Gaul HP, Wallace CJ, Crawley AP. Reverse enhancement of hemorrhagic brain lesions on postcontrast MR: detection with digital image subtraction. AJNR Am J Neuroradiol, 17(9), 1675-1680 (1996).

69. Radiology ACo. ACR-SPR-SSR Practice Parameter for the Performance and Interpretatio of Magnetic Resonance Imaging (MRI) of Bone and Soft Tissue Tumors (Resolution 5). The American College of Radiology (Eds) (2015)

70. Chappell R, Miranpuri SS, Mehta MP. Dimension in defining tumor response. J Clin Oncol, 16(3), 1234 (1998).

71. Ammirati M, Vick N, Liao YL, Ciric I, Mikhael M. Effect of the extent of surgical resection on survival and quality of life in patients with supratentorial glioblastomas and anaplastic astrocytomas. Neurosurgery, 21(2), 201-206 (1987).

72. Curran WJ, Jr., Scott CB, Horton J et al. Recursive partitioning analysis of prognostic factors in three radiation therapy oncology group malignant glioma trials. J Natl Cancer Inst, 85(9), 704-710 (1993).

73. Lacroix M, Abi-Said D, Fourney DR et al. A multivariate analysis of 416 patients with glioblastoma multiforme: prognosis, extent of resection, and survival. J Neurosurg, 95(2), 190-198 (2001).

74. Laws ER, Parney IF, Huang W et al. Survival following surgery and prognostic factors for recently diagnosed malignant glioma: data from the Glioma Outcomes Project. J Neurosurg, 99(3), 467-473 (2003).

75. McGirt MJ, Chaichana KL, Gathinji M et al. Independent association of extent of resection with survival in patients with malignant brain astrocytoma. J Neurosurg, 110(1), 156-162 (2009).

76. Bauchet L, Mathieu-Daude H, Fabbro-Peray P et al. Oncological patterns of care and outcome for 952 patients with newly diagnosed glioblastoma in 2004. Neuro Oncol, 12(7), 725-735 (2010).

77. Li J, Wang M, Won M et al. Validation and simplification of the Radiation Therapy Oncology Group recursive partitioning analysis classification for glioblastoma. Int J Radiat Oncol Biol Phys, 81(3), 623-630 (2011)

78. Sanai N, Polley MY, McDermott MW, Parsa AT, Berger MS. An extent of resection threshold for newly diagnosed glioblastomas. $J$ Neurosurg, 115(1), 3-8 (2011).

79. Chaichana KL, Jusue-Torres I, Navarro-Ramirez R et al. Establishing percent resection and residual volume thresholds affecting survival and recurrence for patients with newly diagnosed intracranial glioblastoma. Neuro Oncol, 16(1), 113-122 (2014).

80. Oppenlander ME, Wolf AB, Snyder LA et al. An extent of resection threshold for recurrent glioblastoma and its risk for neurological morbidity. J Neurosurg, 120(4), 846-853 (2014).

81. Zinn PO, Colen RR, Kasper EM, Burkhardt JK. Extent of resection and radiotherapy in GBM: A 1973 to 2007 surveillance, epidemiology and end results analysis of 21,783 patients. Int J Oncol, 42(3), 929-934 (2013).

82. Pan IW, Ferguson SD, Lam S. Patient and treatment factors associated with survival among adult glioblastoma patients: A USA population-based study from 2000-2010. J Clin Neurosci, 22(10), 1575-1581 (2015).

83. Chaichana KL, Cabrera-Aldana EE, Jusue-Torres I et al. When gross total resection of a glioblastoma is possible, how much resection should be achieved? World Neurosurg, 82(1-2), e257-265 (2014).

84. Grabowski MM, Recinos PF, Nowacki AS et al. Residual tumor volume versus extent of resection: predictors of survival after surgery for glioblastoma. J Neurosurg, 121(5), 1115-1123 (2014).

85. Rostomily RC, Spence AM, Duong D, McCormick K, Bland M, Berger MS. Multimodality management of recurrent adult malignant gliomas: results of a phase II multiagent chemotherapy study and analysis of cytoreductive surgery. Neurosurgery, 35(3), 378388; discussion 388 (1994).

86. Jeremic B, Shibamoto Y, Grujicic D et al. Pre-irradiation carboplatin and etoposide and accelerated hyperfractionated radiation therapy in patients with high-grade astrocytomas: a phase II study. Radiother Oncol, 51(1), 27-33 (1999).

87. Hauch H, Sajedi M, Wolff JE. Treatment arms summarizing analysis of 220 high-grade glioma studies. Anticancer Res, 25(5), 35853590 (2005).

88. Andreou J, George AE, Wise A et al. CT prognostic criteria of survival after malignant glioma surgery. AJNR Am J Neuroradiol, 4(3), 488-490 (1983).

89. Wood JR, Green SB, Shapiro WR. The prognostic importance of tumor size in malignant gliomas: a computed tomographic scan 
study by the Brain Tumor Cooperative Group. J Clin Oncol, 6(2), 338-343 (1988).

90. Vecht CJ, Avezaat CJ, van Putten WL, Eijkenboom WM, Stefanko SZ. The influence of the extent of surgery on the neurological function and survival in malignant glioma. A retrospective analysis in 243 patients. J Neurol Neurosurg Psychiatry, 53(6), 466-471 (1990).

91. Albert FK, Forsting M, Sartor K, Adams HP, Kunze S. Early postoperative magnetic resonance imaging after resection of malignant glioma: objective evaluation of residual tumor and its influence on regrowth and prognosis. Neurosurgery, 34(1), 45-60; discussion 60-41 (1994).

92. Ho J, Ondos J, Ning $\mathrm{H}$ et al. Chemoirradiation for glioblastoma multiforme: the national cancer institute experience. PLoS One, 8(8), e70745 (2013).

93. Ellingson BM, Kim E, Woodworth DC et al. Diffusion MRI quality control and functional diffusion map results in ACRIN 6677/RTOG 0625: a multicenter, randomized, phase II trial of bevacizumab and chemotherapy in recurrent glioblastoma. Int J Oncol, 46(5), 18831892 (2015).
94. Ellingson BM, Wen PY, van den Bent MJ, Cloughesy TF. Pros and cons of current brain tumor imaging. Neuro Oncol, 16 Supp1 7, vii211 (2014).

95. Ellingson BM, Garcia J, Revil C et al. Residual tumor volume and change in tumor volume during adjuvant therapy predict long-term survival in AVAglio: Phase 3 newly diagnosed glioblastoma patients treated with radiation, temozolomide, and bevacizumab or placebo. J Clin Oncol, 34(suppl; abstr 2021) (2016).

96. James K, Eisenhauer E, Christian $\mathrm{M}$ et al. Measuring response in solid tumors: unidimensional versus bidimensional measurement. $J$ Natl Cancer Inst, 91(6), 523-528 (1999).

97. Reardon DA, Ballman KV, Buckner JC, Chang SM, Ellingson BM. Impact of imaging measurements on response assessment in glioblastoma clinical trials. Neuro Oncol, 16 Suppl 7, vii2435 (2014).

98. Lescher S, Schniewindt S, Jurcoane A, Senft C, Hattingen E. Time window for postoperative reactive enhancement after resection of brain tumors: less than 72 hours. Neurosurg Focus, 37(6), E3 (2014). 